\title{
Article
}

\section{Heritability of Curve Patterns in Oral Glucose Tolerance Test}

\author{
Christine Dalgård ${ }^{1}$, Soren Möller² and Kirsten O. Kyvik ${ }^{3}$ \\ ${ }^{1}$ Department of Public Health, Environmental Medicine and The Danish Twin Registry, University of Southern Denmark, Odense C, Denmark, ${ }^{2}$ Odense Patient \\ Data Explorative Network (OPEN), Odense University Hospital and Department of Clinical Research, University of Southern Denmark, Odense C, Denmark and \\ ${ }^{3}$ Department of Clinical Research and The Danish Twin Registry, University of Southern Denmark and Odense Patient Data Explorative Network (OPEN), Odense \\ University Hospital, Odense C, Denmark
}

\begin{abstract}
Type 2 diabetes, which is caused by both genetic and environmental factors, may be diagnosed using the oral glucose tolerance test (OGTT). Recent studies demonstrated specific patterns in glucose curves during OGTT associated with cardiometabolic risk profiles. As the relative contribution of genetic and environmental influences on glucose curve patterns is unknown, we aimed to investigate the heritability of these patterns. We studied twins from the Danish GEMINAKAR cohort aged 18-67 years and free from diabetes at baseline during 1997-2000; glucose concentrations were measured three times during a 2-h OGTT. Heterogeneity of the glucose response during OGTT was examined with latent class mixed-effects models, evaluating goodness of fit by Bayes information criterion. The genetic influence on curve patterns was estimated using quantitative genetic modeling based on linear structural equations. Overall, 1455 twins (41\% monozygotic) had valid glucose concentrations measured from the OGTT, and four latent classes with different glucose response patterns were identified. Statistical modeling demonstrated genetic influence for belonging to a specific class or not, with heritability estimated to be between $45 \%$ and $67 \%$. During $~ 12$ years of follow-up, the four classes were each associated with different incidence of type 2 diabetes. Hence, glucose response curve patterns associated with type 2 diabetes risk appear to be moderately to highly heritable.
\end{abstract}

Keywords: oral glucose tolerance test; latent class modeling; type 2 diabetes; twins; genetic variation

(Received 19 June 2019; accepted 4 December 2019; First Published online 21 February 2020)

Type 2 diabetes is a multicomplex, heterogenic disease with a pathophysiology characterized by insulin resistance and $\beta$-cell dysfunction (Kahn et al., 2014). Diabetes is diagnosed using specific criteria for plasma glucose (PG) concentrations either during fasting [fasting plasma glucose (FPG) $\geq 7 \mathrm{mmol} / \mathrm{l}$ ] or at $2 \mathrm{~h}$ (PG $\geq 11.1 \mathrm{mmol} / \mathrm{l})$ during an oral glucose tolerance test (OGTT; American Diabetes Association, 2019). However, the change in PG concentrations from healthy to the development of type 2 diabetes is a continuous process, where increases in glucose concentrations even within the normal range are found to relate to long-term risk for type 2 diabetes (Faerch et al., 2013; Hulsegge et al., 2017; Malmstrom et al., 2018; Tabak et al., 2009). Recent studies have demonstrated that not only the FPG and the 2-h PG values indicate the future risk of diabetes but also the intermediate glucose concentrations during the OGTT, and hence the shape of the glucose response curve may predict the risk in subjects initially free from diabetes (Abdul-Ghani et al., 2010; Hayashi et al., 2013; Hulman, Gujral et al., 2017; Hulman, Simmons, Vistisen et al., 2017; Hulman et al., 2018). Hulman and colleagues have shown that there is considerable heterogeneity in the glucose response curves, and they demonstrated using latent class modeling that four or five specific latent classes of OGTT curve pattern appear, each associated with specific cardiometabolic

Author for correspondence: Christine Dalgård, Email: cdalgaard@health.sdu.dk Cite this article: Dalgård C, Möller S, and Kyvik KO. (2020) Heritability of Curve Patterns in Oral Glucose Tolerance Test. Twin Research and Human Genetics 23: 39-44, https://doi.org/10.1017/thg.2020.3 risk profiles as well as future diabetes risk (Hulman, Gujral et al., 2017; Hulman, Simmons, Vistisen et al., 2017; Hulman et al., 2018). However, it is somehow uncertain what affects a person's classification or 'belonging' to one of the classes of the OGTT curve patterns, although it is also clearly related to age, body mass index (BMI) and other cardiometabolic risk factors as demonstrated in the previous studies (Hulman, Gujral et al., 2017; Hulman, Simmons, Vistisen et al., 2017; Hulman et al., 2018).

Nevertheless, genetic variation has been reported associated with both fasting glucose (Dupuis et al., 2010; Morris et al., 2012) and 2-h PG after OGTT (Saxena et al., 2010). Also, we and others have demonstrated using twin studies that fasting as well as 2-h glucose levels are heritable, ranging from $12 \%$ to $62 \%$ (Liu et al., 2009; Schousboe et al., 2003), with a common set of genetic factors influencing them both (Liu et al., 2009).

We therefore speculated whether genetic variation, to some extent, could explain the heterogenic glucose response curve patterns and thus aimed to investigate the relative contribution of genetic and environmental factors for OGTT curve patterns using a twin design.

\section{Materials and Methods}

\section{Study Population}

The GEMINAKAR cohort was initiated in 1997 as an observational, prospective twin cohort study of 756 complete twin-pairs, aged 18-67 years, recruited from the nationwide populationbased Danish Twin Registry during 1997-2000 as previously 
described (Pedersen et al., 2019; Schousboe et al., 2003). To be included in the GEMINAKAR cohort, both twins in a pair had to be willing to participate. Also, the twins had to be without known diabetes or cardiovascular disease, be able to make a progressive maximal bicycle test and not be pregnant or breastfeeding.

The participants were followed up between 2010 and 2012, irrespective of whether both twins in a pair were able to or wanted to participate in the follow-up. A total of 1139 ( 79\%) gave consent to participate with clinical examination in the follow-up.

At baseline, the participants underwent a standardized OGTT described below. Furthermore, at both baseline and follow-up, the participants went through a clinical examination, including anthropometric measurements and fasting blood sampling, and answered questionnaires regarding dietary habits, lifestyle and socioeconomic factors. Finally, the participants reported whether they were diagnosed with diabetes mellitus and cardiovascular disease by their physician.

For the current investigation, we excluded those with previously diagnosed diabetes (self-reporting a physician diagnosis of diabetes in questionnaire) or screen-detected diabetes (blood glucose $\geq$ $6.1 \mathrm{mmol} / \mathrm{l}$ or 2 -h blood glucose $\geq 11.1 \mathrm{mmol} / \mathrm{l}$; Alberti \& Zimmet, 1998) based on the OGTT. Our study population was thus limited to 1455 twins who had valid data from the OGTT and were without diabetes at baseline.

All participants gave written informed consent both at baseline and follow-up. The study was approved by the regional Health Research Ethics Committee of the Region of Southern Denmark (baseline, S-VF-19970271; follow-up, S-20090065) and the SDU Research \& Innovation Organisation, in compliance with the General Data Protection Regulation (17/50526).

\section{Assessment of OGTT}

At baseline, the twins in a pair were examined at the same day. They underwent a standardized 2-h 75-g OGTT in the morning between 8:00 and 11:00 am after a 10- to 12-h overnight fast (Schousboe et al., 2003). After $15 \mathrm{~min}$ of rest, a capillary blood sample was drawn immediately before ingestion of the glucose solution, and two additional capillary blood samples were drawn 30 and 120 min later. Peripheral venous blood samples for insulin measurements were drawn at the same time points.

\section{Assessment of Type 2 Diabetes}

At follow-up, incident type 2 diabetes was defined as self-reported diabetes, FPG $\geq 7.0 \mathrm{mmol} / \mathrm{l}, \mathrm{HbA} 1 \mathrm{c} \geq 48 \mathrm{mmol} / \mathrm{mol}(6.5 \%)$ or self-reported use of antidiabetic medicine.

\section{Assessment of Covariates}

At both baseline and follow-up, the clinical examination included measurements of weight in light clothing (to the nearest $.1 \mathrm{~kg}$ ) and height without shoes (to the nearest $.1 \mathrm{~cm}$ ), and BMI was calculated as weight (in $\mathrm{kg}$ ) divided by height squared $\left(\mathrm{m}^{2}\right)$. Waist circumference (to the nearest $.1 \mathrm{~cm}$ ) was measured midway between the lowest rib and the iliac crest. Systolic and diastolic blood pressures were measured after 30 min rest using a conventional mercury sphygmomanometer and hands-free stethoscope at the right arm. The average of three blood pressure measurements was used (Benyamin et al., 2007).

At baseline, capillary blood glucose concentrations were measured by the glucose dehydrogenase oxidation method, and serum insulin concentrations were measured using a commercial timeresolved immunofluorometric assay (AutoDelfia; PerkinElmer, Turku, Finland) as reported previously (Schousboe et al., 2003). Cholesterol, high-density lipoproteins (HDLs) and triglycerides were measured on fasting serum samples by a colorimetric method (VITROS; Johnson \& Johnson, New Brunswick, NJ, USA). Lowdensity lipoproteins (LDLs) were calculated (Friedewald formula) by subtracting HDL and $(.45 \times$ trigylceride) from total cholesterol (Benyamin et al., 2007).

At follow-up, peripheral venous blood samples were collected for $\mathrm{HbA1c}$ analysis carried out using a fully automated glycohemoglobin analyzer (Tosoh G8 equipment, Tosoh Corporation, Bioscience Division; Tosoh G8, Tokyo, Japan; Nielsen et al., 2014). Also, FPG was determined using a hexokinase/G-6-PDH principle (Integra 700/Roche equipment).

Zygosity of the twins was determined by nine polymorphic DNA-based microsatellite markers with the PE Applied Biosystems AmpFISTR Profiler Plus Kit (Perkin Elmer, Foster City, CA, USA) (Schousboe et al., 2003).

\section{Statistical Analysis}

Baseline data are expressed as median (25th-75th percentiles) or frequency (\%). We tested differences between classes using the Kruskal-Wallis test for continuous variables and chi-square for categorical variables. Heterogeneity of glucose response during OGTT was modeled with latent class mixed-effects models, which for a predetermined number of latent classes identifies a grouping of observations, resulting in the most homogenous trajectories of glucose response within each group, following strategies applied in similar studies on singletons (Hulman, Gujral et al., 2017). We investigated 2-7 latent variables. For more than seven latent variables, the sample size was not sufficient to achieve model convergence. We evaluated goodness of fit by Bayes information criterion and applied the model for a varying number of latent classes. We allowed age and sex of the twin individuals to influence the class membership and decided not to include the twin structure to avoid overfitting with respect to the following twin analyses.

We used the classical twin models to determine the contribution of genetic and environmental factors to the variance in class belonging. These models make use of the difference in genetic relatedness between monozygotic (MZ) twins (sharing $~ 100 \%$ of their segregating genes) and the dizygotic (DZ) twins (sharing on average $50 \%$ of their segregating genes); hence, any greater concordance between MZ twins than DZ twins is assumed to be related to this genetic sharing. The modeling is based on structural equations to estimate the best fit of the proportion of variance on individual class membership that can be explained by variation in additive genetic factors (A), dominant genetic factors (D), common/shared environmental factors (C) and unique environmental factors $(\mathrm{E})$. The heritability is the proportional contribution of genetic variance $(A+D)$ to the total phenotypic variance $(\mathrm{A}+\mathrm{C}+\mathrm{D}+\mathrm{E})$. We investigated $\mathrm{AE}, \mathrm{DE}, \mathrm{CE}, \mathrm{ACE}, \mathrm{ADE}$ and DCE models and decided on the preferred model on the basis of optimal Akaike information criterion. In the models, we adjusted the marginal (individual) effect of each twin for the persons own sex and age.

Finally, we used logistic regression to examine the association between class belonging and diabetes at follow-up. We adjusted for age, sex, smoking status, cholesterol, HDL, BMI, waist circumference and systolic and diastolic blood pressure and used robust standard errors to account for dependency within twin-pairs. 
Table 1. Characteristics of study participants stratified according to glucose response latent class curve

\begin{tabular}{|c|c|c|c|c|c|c|}
\hline$n(\%)$ & $\begin{array}{c}\text { Overall } \\
1455\end{array}$ & $\begin{array}{c}\text { Class } 1 \\
554(37 \%)\end{array}$ & $\begin{array}{c}\text { Class } 2 \\
549(36 \%)\end{array}$ & $\begin{array}{c}\text { Class } 3 \\
244(16 \%)\end{array}$ & $\begin{array}{c}\text { Class } 4 \\
108(7 \%)\end{array}$ & $p$ Value $^{\star}$ \\
\hline \multicolumn{7}{|c|}{ Demographic and cardiometabolic characteristics } \\
\hline Female, $n(\%)$ & $759(52.2)$ & $311(56.1)$ & $364(66.3)$ & $36(14.8)$ & $48(44.4)$ & $<.001$ \\
\hline Smoker, $n(\%)$ & $476(32.7)$ & $153(27.6)$ & $157(28.6)$ & $119(48.8)$ & $47(43.5)$ & $<.001$ \\
\hline Cholesterol (mmol/l) & $5.3(4.6-6.1)$ & $4.9(4.3-5.6)$ & $5.5(4.8-6.3)$ & $5.4(4.6-6.1)$ & $6.0(5.3-6.9)$ & $<.0001$ \\
\hline $\mathrm{HDL}(\mathrm{mmol} / \mathrm{l})$ & $1.5(1.2-1.7)$ & $1.4(1.2-1.7)$ & $1.5(1.2-1.8)$ & $1.4(1.1-1.7)$ & $1.5(1.3-1.8)$ & .0003 \\
\hline $\mathrm{LDL}(\mathrm{mmol} / \mathrm{l})$ & $3.2(2.6-3.9)$ & $2.9(2.4-3.6)$ & $3.4(2.7-4.1)$ & $3.3(2.7-4.0)$ & $3.8(3.0-4.4)$ & $<.0001$ \\
\hline Triglyceride (mmol/l) & $1.1(.9-1.5)$ & $1.0(.8-1.3)$ & $1.2(.9-1.6)$ & $1.2(.9-1.6)$ & $1.4(1.0-2.0)$ & $<.0001$ \\
\hline Fasting glucose (mmol/l) & $4.7(4.4-5.0)$ & $4.5(4.3-4.8)$ & $4.8(4.5-5.1)$ & $4.8(4.6-5.1)$ & $5.3(4.9-5.7)$ & $<.0001$ \\
\hline 30-min glucose $(\mathrm{mmol} / \mathrm{l})$ & $8.4(7.5-9.3)$ & $7.1(6.6-7.7)$ & $8.8(8.3-9.4)$ & $9.1(8.6-9.9)$ & $11.1(10.6-11.9)$ & $<.0001$ \\
\hline 2-h glucose $(\mathrm{mmol} / \mathrm{l})$ & $6.0(5.4-6.7)$ & $5.6(5.2-6.1)$ & $6.7(6.3-7.2)$ & $5.0(4.3-5.4)$ & $7.4(6.7-8.4)$ & $<.0001$ \\
\hline Fasting insulin $(\mathrm{pmol} / \mathrm{l})$ & $33(24-46)$ & $32(24-44)$ & $35(25-46)$ & $31(22-43)$ & $38(23-54)$ & $<.0331$ \\
\hline 30-min insulin (pmol/l) & $269(188-386)$ & $242(178-334)$ & 273 (195-399) & $301(208-420)$ & $305(185-426)$ & $<.0001$ \\
\hline 2-h insulin (pmol/l) & $134(84-210)$ & $120(80-172)$ & $175(119-254)$ & $66(39-107)$ & $226(140-360)$ & $<.0001$ \\
\hline
\end{tabular}

Note: Data are expressed as median (25th-75th percentiles) or frequency (\%). ${ }^{*} p$ values from $\chi^{2}$ analyses for categorical variables or Kruskal-Wallis test for continuous variables.

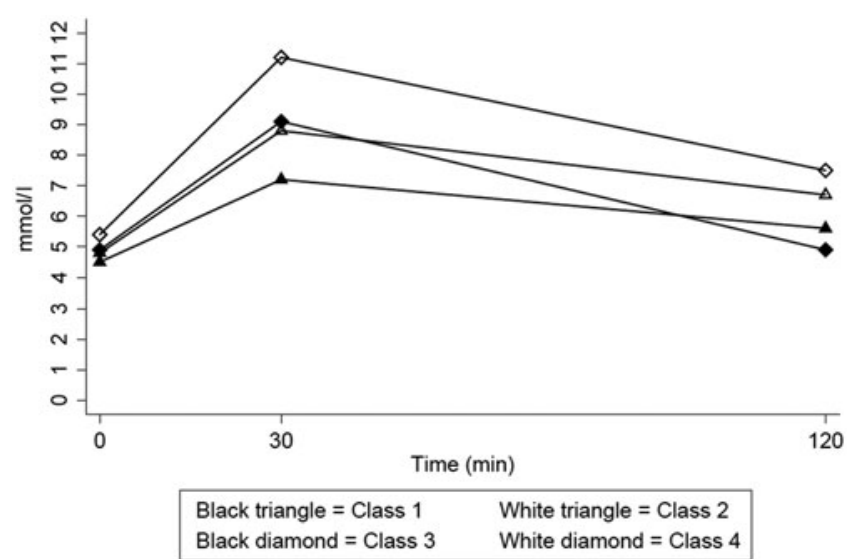

Fig. 1. Heterogenous blood glucose response curves during an OGTT among 1455 twins.

We decided not to carry out analyses stratified by age or sex, due to the limited sample size.

The statistical modeling was performed in Stata (Release 15. College Station, TX, USA: StataCorp LLC) and RStudio version 1.0.143 (RStudio, Inc., Boston, MA).

\section{Results}

The baseline characteristics of the study population are demonstrated in Table 1. In total, 1455 twins, consisting of 708 complete pairs (42\% MZ) and 39 singular twins (33\% MZ), had valid glucose concentrations measured from the OGTT (>99\% with all three measures) and were without diabetes at baseline. We identified four latent classes with different glucose response patterns comprising between $7 \%(n=108$, Class 4$)$ and $37 \%(n=554$, Class 1) of the cohort (Figure 1).

For glucose, Class 1 experienced the lowest values, Class 2 moderate values, and Class 4 the highest glucose values at all three time points. However, Class 3, although characterized by third highest glucose values at time zero (fasting) and $30 \mathrm{~min}$, had the lowest glucose values at $2 \mathrm{~h}$. All classes had lower 2 -h values than at $30 \mathrm{~min}$, but only Class 3 returned to fasting levels (Figure 1, Table 1).

For insulin, all four classes experienced almost same levels at fasting; however, Class 1 experienced the lowest increase at time $30 \mathrm{~min}$ and second lowest at time $2 \mathrm{~h}$. Class 3 had the highest insulin response at $30 \mathrm{~min}$ and the steepest decline to the 2 -h values, compared with the three other classes. Class 4 experienced a high and the highest levels at the following time points, respectively, compared with the other classes. All classes had lower 2-h values than at $30 \mathrm{~min}$, but none of them returned to fasting levels (Table 1).

The four classes differed in their baseline characteristics. In general, Class 4 had the highest values of the cardiometabolic risk factors compared with the three other classes, except for HDL and waist circumference. Class 4 also had the highest mean age, 49 years compared with 34-40 years for the other three classes. Class 3 had the lowest proportion of women, $\sim 15 \%$ compared with $44-66 \%$, and the highest proportion of smokers, $49 \%$ compared with $28-44 \%$ for the other three classes (Table 1 , all $p$ values <.05). 
Table 2. Casewise concordance rates showing probability of belonging to the same predicted latent class as the co-twin and best-fitting models to estimate the quantitative contribution of environmental and genetic factors on variance within each class

\begin{tabular}{|c|c|c|c|c|c|c|c|c|c|c|c|c|}
\hline \multirow[b]{2}{*}{ Class } & \multirow[b]{2}{*}{ Twins $(n)$} & \multicolumn{2}{|c|}{$\begin{array}{c}\text { Twins in } 372 \\
\text { concordant } \\
\text { pairs }(n)\end{array}$} & \multicolumn{2}{|c|}{$\begin{array}{c}\text { Twins in } 336 \\
\text { discordant } \\
\text { pairs }(n)^{\mathrm{a}}\end{array}$} & \multirow[b]{2}{*}{ Marginal $^{\mathrm{b}}(95 \% \mathrm{Cl})$} & \multicolumn{2}{|c|}{$\begin{array}{l}\text { Casewise concordance } \\
\qquad(95 \% \mathrm{Cl})\end{array}$} & \multicolumn{4}{|c|}{$\begin{array}{l}\text { Parameter estimates }{ }^{c} \\
(95 \% \mathrm{Cl})\end{array}$} \\
\hline & & $M Z$ & $\mathrm{DZ}$ & $M Z$ & $\mathrm{DZ}$ & & $M Z$ & $\mathrm{DZ}$ & A & D & C & $E$ \\
\hline 1 & 554 & 166 & 166 & 72 & 150 & $.38[.35, .41]$ & $.70[.63, .76]$ & $.55[.49, .61]$ & $.67[.55, .78]$ & NA & NA & $.33[.22, .45]$ \\
\hline 2 & 549 & 124 & 166 & 94 & 165 & $.38[.35, .41]$ & $.59[.51, .66]$ & $.50[.43, .56]$ & $.45[.31, .60]$ & NA & NA & $.55[.40, .69]$ \\
\hline 3 & 244 & 46 & 44 & 57 & 97 & $.17[.15, .19]$ & $.45[.35, .57]$ & $.33[.24, .43]$ & $.48[.29, .66]$ & NA & NA & $.52[.34, .71]$ \\
\hline 4 & 108 & 16 & 16 & 28 & 48 & $.075[.06, .09]$ & $.42[.25, .61]$ & $.28[.16, .45]$ & $.63[.42, .84]$ & NA & NA & $.37[.16, .58]$ \\
\hline
\end{tabular}

${ }^{a}$ Total number also includes 39 twins without their co-twin in the present study.

${ }^{b}$ Marginal indicates the expected class concordance for two unrelated twins in the cohort.

${ }^{\mathrm{C}} \mathrm{A}$ : additive genetic effect, D: dominant genetic effect, C: shared environmental effect, E: unique (nonshared) environmental effect (including error), all reported with $95 \% \mathrm{Cl}$.

There were marked differences in the various cardiometabolic risk factors between classes; Class 3 mean values were between Classes 1 and 2 for most of the risk factors except for waist circumference, BMI and systolic blood pressure.

For $53 \%$ of the pairs ( 372 of 708 complete pairs), both twins in a pair were assigned into the same class, consisting of a concordance of $60 \%$ (176 of 295) in MZ pairs and 47\% (196 of 413) in DZ pairs. In comparison, the expected class concordance for two unrelated twins in the cohort would be only $32 \%$. The observed casewise concordances for the individual classes varied between $42-70 \%$ in $\mathrm{MZ}$ twins and $28-55 \%$ in $\mathrm{DZ}$ twins.

We fitted different models for each of the four classes. Generally, the best-fitting models were the reduced AE models, with estimates indicating that the additive genetic component (the heritability) explained between $45 \%$ (95\% confidence interval [CI] $[31 \%, 60 \%)$ and $67 \%(95 \%$ CI $[55 \%, 78 \%])$ and the unique environmental component explained between 33\% (95\% CI $[22 \%, 45 \%])$ and $55 \%(95 \%$ CI $[40 \%, 71 \%])$ of the total variation of belonging to a specific class (Table 2).

At follow-up during 2010-2012 ( 12 years after first intake), $1139(79 \%)$ twins were participating and examined clinically; $3.9 \%$ were found to have type 2 diabetes. Among these, 1098 had been categorized by the glucose curve pattern class at baseline. Participants in Class 4 had a more than five-fold higher incidence of type 2 diabetes than the highest of the three other classes (20\% vs. .2-3.5\%). A clear association was observed between class belonging and diabetes. After adjustment for potential confounders, the odds ratio of diabetes was $>70$ times higher among participants classified into Class 4 (OR=75.5; 95\% CI $[8.3,689.0], p<.001)$ compared with participants classified into Class 1. The large magnitude of this OR is biological implausible and caused by limited number of cases in the classes; however, a strong association, albeit most probably with less extreme true $\mathrm{OR}$, with diabetes was robust also after adjustments.

\section{Discussion}

In the present study, we used a latent class analysis to identify four separate classes of glucose response to an OGTT, each with very different incidence of type 2 diabetes $\sim 12$ years later. Furthermore, we determined the relative environmental and genetic contribution to the variation in belonging to one of the classes. The influence of unique environmental factors of 33-55\% illustrates the preventative potential of type 2 diabetes.
We have used the same data-driven method to identify latent classes among subjects free from diabetes at baseline as Hulman and colleagues did in three recent studies (Hulman, Gujral et al., 2017; Hulman, Simmons, Vistisen et al., 2017; Hulman et al., 2018); our study supports these studies both regarding curve shapes and the risk of incident type 2 diabetes according to class belonging. This is notable, as the four studies have differences among population characteristics. For instance, one of Hulman et al.'s studies was performed in an Asian Indian population (Hulman, Simmons, Brunner et al., 2017), and ours in a European population, mean ages ranged from $37-38$ years to $49-50$ years of age, and baseline fasting glucose (FG) ranged from 4.7 to $5.4 \mathrm{mmol} / \mathrm{l}$.

Also, the long-term risk of type 2 diabetes associated with these patterns observed in the present study supports what has been demonstrated in the previous studies: the class with high/the highest blood glucose values at all three time points has the highest risk of diabetes. The study thus lends support to previous studies demonstrating that using both the FPG and glucose concentrations during the OGTT may improve the identification of subjects who are at increased risk for type 2 diabetes (Abdul-Ghani et al., 2010; Alyass et al., 2015; Hayashi et al., 2013). The differences in risk between classes could be a result of the stage or trajectory for the development of type 2 diabetes, for example, the higher the mean age of the class, the higher the risk of type 2 diabetes of the class. It is well known that age is an important risk factor for type 2 diabetes, but still, we observed a much higher prevalence of diabetes in Class 4 than could be anticipated due to age alone (Guariguata et al., 2014; Wild et al., 2004). Also, the two articles by Hulman et al. and our results demonstrated that type 2 diabetes risk by class membership was independent of differences in wellknown risk factors, including age, sex and waist circumference (Hulman, Gujral et al., 2017; Hulman et al., 2018). In further support of this, our results demonstrated that even with same sex, age and genotype, twins in a pair may be classified into different classes; for instance, $40 \%$ of the MZ twins in the complete pairs were classified differently.

Our quantitative genetic modeling demonstrated a moderateto-high genetic influence on the probability of belonging to a specific class, given that the co-twin already was in the same class (Table 2). The high, but not full, concordance rate for the MZ twins suggests that also environmental factors influence class belonging. Furthermore, the higher concordance rates for the $\mathrm{MZ}$ twins than the $\mathrm{DZ}$ twins, which again are higher than for two unrelated twins in the cohort, indicate involvement of shared environment in the 
belonging of class. However, the modeling could not demonstrate an ACE model that includes shared environment (i.e., shared family-level environmental factors) but only an AE model; hence, the influence of shared environment is either rather low or nonexisting or there is not enough power to detect. Finally, the models estimated that the influence of genetic factors on belonging to a specific class was moderate to high (heritability varied from $45 \%$ to $67 \%$ ). This demonstrates that, depending on class, environmental factors may have varying influence on belonging to a specific class, ranging from $33 \%$ to $55 \%$ (Table 2).

We were not able to identify other studies that have examined the heritability of the full OGTT curve patterns. But the values agree with heritability estimates for the time-specific PG concentrations during an OGTT found in other studies. Although some studies have found a higher heritability for fasting glucose concentrations than for 2-h glucose concentrations (Almgren et al., 2011), we and others have previously found the opposite (Katoh et al., 2005; Liu et al., 2009; Schousboe et al., 2003). Furthermore, Liu et al. (2009) performed bivariate analyses of fasting and 2-h glucose concentrations and observed heritability estimates very like ours.

As the different curve patterns are associated with different risk of type 2 diabetes, finding the environmental factors that influence the individual class membership would potentially bring new understanding to the development of type 2 diabetes. Also, together with the knowledge on environmental factors, more insight would be gained by analyses of the $\mathrm{G} \times \mathrm{E}$ interaction.

Our study has several strengths, including the twin population recruited from a population-based register and the longitudinal study design. Moreover, the availability of three glucose measurements for each twin and the twin structure allowed us to combine the latent class modeling with classic twin methodology. However, it was not possible to consider the interplay between uncertainties from both methods at the same time, which might influence the CIs reported from the twin models. Previous studies have found sex-specific differences for fasting glucose and 2-h PG heritability, but still with this relatively large sample of twins we could not stratify by sex. Hence, the latent curve patterns are not estimated stratified by sex, and we were therefore not able to discover potential sex-specific differences in the relative contribution of genes and environment for curve patterns. Furthermore, the classical twin model cannot reflect gene-environment interaction with a possible result of an overestimation of environmental contribution to variance (Purcell, 2002). Also, a recent study has demonstrated age-varying heritability estimates of type 2 diabetes (Almgren et al., 2011). It is therefore possible that the same age variation would be present in the OGTT curve patterns as they are related to type 2 diabetes risk. We did not stratify our analyses by age, again due to sample size and potential loss of power. However, the genetic variation of the pattern was of almost the same magnitude in the youngest Class 1 and oldest Class 4 , although there was more than 15 years difference in median age, indicating that age may not be the primary determinant of the total phenotypic variation. Although we used a population-based register for recruitment (Pedersen et al., 2019), the participants were twins, which could limit the generalizability of our findings to singletons. Nevertheless, several studies have found similar prevalences of type 2 diabetes and other diseases in twins and singletons (Christensen et al., 2001; Petersen et al., 2011).

In conclusion, in a nondiabetic population, we identified four classes of distinct glucose response curve patterns and demonstrated that individuals characterized by high $30-\mathrm{min}$ and 2 -h
PG are at increased risk for incident type 2 diabetes. We showed that genetic variation explains a moderate-to-high proportion of variation in class belonging. Finding the genetic as well as the environmental factors that act on these curves may offer insight into potential personalized preventative strategies.

Author contributions. SM analyzed data; $\mathrm{CD}$ analyzed data, wrote the manuscript and has the primary responsibility for the final content. CD and KOK designed the study. All authors interpreted data, reviewed, revised and edited the manuscript for intellectual content, and all authors approved the final version. $\mathrm{CD}$ is the guarantor of this work and, as such, had full access to all the data in the study and takes responsibility for the integrity of the data and the accuracy of the data analyses.

Financial support. The GEMINAKAR cohort was supported by grants from the Danish Medical Research Fund; The Danish Diabetes Association; The NOVO Foundation; The Danish Heart Foundation; Interreg 4a Southern Denmark-Schleswig-KERN funded by the European Regional Development Fund (grant number 57-1.3-10); National Institute of Aging-National Institutes of Health, USA (grant number 95-103-11412); and the Danish Research Council for Health and Disease.

Conflict of interest. None.

\section{References}

Abdul-Ghani, M. A., Lyssenko, V., Tuomi, T., Defronzo, R. A., \& Groop, L. (2010). The shape of plasma glucose concentration curve during OGTT predicts future risk of type 2 diabetes. Diabetes/Metabolism Research and Reviews, 26, 280-286.

Alberti, K. G., \& Zimmet, P. Z. (1998). Definition, diagnosis and classification of diabetes mellitus and its complications. Part 1: Diagnosis and classification of diabetes mellitus provisional report of a WHO consultation. Diabetic Medicine, 15, 539-553.

Almgren, P., Lehtovirta, M., Isomaa, B., Sarelin, L., Taskinen, M. R., Lyssenko, V., \& Groop, L. (2011). Heritability and familiality of type 2 diabetes and related quantitative traits in the Botnia Study. Diabetologia, 54, 2811-2819.

Alyass, A., Almgren, P., Akerlund, M., Dushoff, J., Isomaa, B., Nilsson, P., \& Meyre, D. (2015). Modelling of OGTT curve identifies $1 \mathrm{~h}$ plasma glucose level as a strong predictor of incident type 2 diabetes: Results from two prospective cohorts. Diabetologia, 58, 87-97.

American Diabetes Association (2019). Classification and diagnosis of diabetes: Standards of medical care in diabetes. Diabetes Care, 42, S13-S28.

Benyamin, B., Sorensen, T. I., Schousboe, K., Fenger, M., Visscher, P. M., \& Kyvik, K. O. (2007). Are there common genetic and environmental factors behind the endophenotypes associated with the metabolic syndrome? Diabetologia, 50, 1880-1888.

Christensen, K., Wienke, A., Skytthe, A., Holm, N. V., Vaupel, J. W., \& Yashin, A. I. (2001). Cardiovascular mortality in twins and the fetal origins hypothesis. Twin Research, 4, 344-349.

Dupuis, J., Langenberg, C., Prokopenko, I., Saxena, R., Soranzo, N., Jackson, A. U., \& Pramstaller, P. P. (2010). New genetic loci implicated in fasting glucose homeostasis and their impact on type 2 diabetes risk. Nature Genetics, 42, 105-116.

Faerch, K., Witte, D. R., Tabak, A. G., Perreault, L., Herder, C., Brunner, E. J., \& Vistisen, D. (2013). Trajectories of cardiometabolic risk factors before diagnosis of three subtypes of type 2 diabetes: A post-hoc analysis of the longitudinal Whitehall II cohort study. Lancet Diabetes \& Endocrinology, $1,43-51$.

Guariguata, L., Whiting, D. R., Hambleton, I., Beagley, J., Linnenkamp, U., \& Shaw, J. E. (2014). Global estimates of diabetes prevalence for 2013 and projections for 2035. Diabetes Research and Clinical Practice, 103, 137-149.

Hayashi, T., Boyko, E. J., Sato, K. K., McNeely, M. J., Leonetti, D. L., Kahn, S. E., \& Fujimoto, W. Y. (2013). Patterns of insulin concentration during the OGTT predict the risk of type 2 diabetes in Japanese Americans. Diabetes Care, 36, 1229-1235. 
Hulman, A., Gujral, U. P., Narayan, K. M. V., Pradeepa, R., Mohan, D., Anjana, R. M., \& Witte, D. R. (2017). Glucose patterns during the OGTT and risk of future diabetes in an urban Indian population: The CARRS study. Diabetes Research and Clinical Practice, 126, 192-197.

Hulman, A., Simmons, R. K., Brunner, E. J., Witte, D. R., Faerch, K., Vistisen, D., \& Tabak, A. G. (2017). Trajectories of glycaemia, insulin sensitivity and insulin secretion in South Asian and white individuals before diagnosis of type 2 diabetes: A longitudinal analysis from the Whitehall II cohort study. Diabetologia, 60, 1252-1260.

Hulman, A., Simmons, R. K., Vistisen, D., Tabak, A. G., Dekker, J. M., Alssema, M., \& Faerch, K. (2017). Heterogeneity in glucose response curves during an oral glucose tolerance test and associated cardiometabolic risk. Endocrine, 55, 427-434.

Hulman, A., Vistisen, D., Glumer, C., Bergman, M., Witte, D. R., \& Faerch, K. (2018). Glucose patterns during an oral glucose tolerance test and associations with future diabetes, cardiovascular disease and all-cause mortality rate. Diabetologia, 61, 101-107.

Hulsegge, G., Spijkerman, A. M. W., van der Schouw, Y. T., Bakker, S. J. L., Gansevoort, R. T., Smit, H. A., \& Verschuren, W. M. M. (2017). Trajectories of metabolic risk factors and biochemical markers prior to the onset of type 2 diabetes: The population-based longitudinal Doetinchem study. Nutrition \& Diabetes, 7, e270.

Kahn, S. E., Cooper, M. E., \& Del Prato, S. (2014). Pathophysiology and treatment of type 2 diabetes: Perspectives on the past, present, and future. Lancet, 383, 1068-1083.

Katoh, S., Lehtovirta, M., Kaprio, J., Harjutsalo, V., Koskenvuo, M., Eriksson, J., \& Tuomilehto, J. (2005). Genetic and environmental effects on fasting and postchallenge plasma glucose and serum insulin values in Finnish twins. Journal of Clinical Endocrinology and Metabolism, 90, 2642-2647.

Liu, G. F., Riese, H., Spector, T. D., Mangino, M., O’Dell, S. D., Stolk, R. P., \& Snieder, H. (2009). Bivariate genetic modelling of the response to an oral glucose tolerance challenge: A gene $\times$ environment interaction approach. Diabetologia, 52, 1048-1055.

Malmstrom, H., Walldius, G., Carlsson, S., Grill, V., Jungner, I., Gudbjornsdottir, S., \& Hammar, N. (2018). Elevations of metabolic risk factors 20 years or more before diagnosis of type 2 diabetes: Experience from the AMORIS study. Diabetes, Obesity and Metabolism, 20, 1419-1426.

Morris, A. P., Voight, B. F., Teslovich, T. M., Ferreira, T., Segre, A. V., Steinthorsdottir, V., \& McCarthy, M. I. (2012). Large-scale association analysis provides insights into the genetic architecture and pathophysiology of type 2 diabetes. Nature Genetics, 44, 981-990.

Nielsen, A. A., Petersen, P. H., Green, A., Christensen, C., Christensen, H., \& Brandslund, I. (2014). Changing from glucose to HbAlc for diabetes diagnosis: Predictive values of one test and importance of analytical bias and imprecision. Clinical Chemistry and Laboratory Medicine, 52, 1069-1077.

Pedersen, D. A., Larsen, L. A., Nygaard, M., Mengel-From, J., McGue, M., Dalgard, C., \& Christensen, K. (2019). The Danish Twin Registry: An updated overview. Twin Research and Human Genetics, 1-9. Epub ahead of publication.

Petersen, I., Nielsen, M. M., Beck-Nielsen, H., \& Christensen, K. (2011). No evidence of a higher 10 year period prevalence of diabetes among 77,885 twins compared with 215,264 singletons from the Danish birth cohorts 1910-1989. Diabetologia, 54, 2016-2024.

Purcell, S. (2002). Variance components models for gene-environment interaction in twin analysis. Twin Research, 5, 554-571.

Saxena, R., Hivert, M. F., Langenberg, C., Tanaka, T., Pankow, J. S., Vollenweider, P., \& Watanabe, R. M. (2010). Genetic variation in GIPR influences the glucose and insulin responses to an oral glucose challenge. Nature Genetics, 42, 142-148.

Schousboe, K., Visscher, P. M., Henriksen, J. E., Hopper, J. L., Sorensen, T. I., \& Kyvik, K. O. (2003). Twin study of genetic and environmental influences on glucose tolerance and indices of insulin sensitivity and secretion. Diabetologia, 46, 1276-1283.

Tabak, A. G., Jokela, M., Akbaraly, T. N., Brunner, E. J., Kivimaki, M., \& Witte, D. R. (2009). Trajectories of glycaemia, insulin sensitivity, and insulin secretion before diagnosis of type 2 diabetes: An analysis from the Whitehall II study. Lancet, 373, 2215-2221.

Wild, S., Roglic, G., Green, A., Sicree, R., \& King, H. (2004). Global prevalence of diabetes: Estimates for the year 2000 and projections for 2030 Diabetes Care, 27, 1047-1053. 